\title{
Balanced Networks of Spiking Neurons with Spatially Dependent Recurrent Connections
}

\author{
Robert Rosenbaum and Brent Doiron \\ Department of Mathematics, University of Pittsburgh, Pittsburgh, Pennsylvania 15260, USA \\ and Center for the Neural Basis of Cognition, Pittsburgh, Pennsylvania 15213, USA \\ (Received 27 August 2013; revised manuscript received 14 February 2014; published 28 May 2014) \\ Networks of model neurons with balanced recurrent excitation and inhibition capture the irregular and \\ asynchronous spiking activity reported in cortex. While mean-field theories of spatially homogeneous \\ balanced networks are well understood, a mean-field analysis of spatially heterogeneous balanced networks \\ has not been fully developed. We extend the analysis of balanced networks to include a connection \\ probability that depends on the spatial separation between neurons. In the continuum limit, we derive that \\ stable, balanced firing rate solutions require that the spatial spread of external inputs be broader than that of \\ recurrent excitation, which in turn must be broader than or equal to that of recurrent inhibition. Notably, this \\ implies that network models with broad recurrent inhibition are inconsistent with the balanced state. For \\ finite size networks, we investigate the pattern-forming dynamics arising when balanced conditions are not \\ satisfied. Our study highlights the new challenges that balanced networks pose for the spatiotemporal \\ dynamics of complex systems.
}

DOI: 10.1103/PhysRevX.4.021039

\section{INTRODUCTION}

The study of spatiotemporal dynamics and variability in complex systems is at the interface of the physical, chemical, biological, and social sciences [1,2]. A central question in many disciplines is, how does high-dimensional, microscopic behavior scale to define the macroscopic dynamics? The answer necessarily depends on the architecture of interactions. In many cases of interest, the complexity of this architecture makes elucidating system-wide dynamics a challenge.

In the neurosciences, a long-standing topic of interest is the significant variability in neuron spike train responses [3-5]. Models of cortical networks capture this high variability when recurrent excitatory and inhibitory inputs are balanced. Such "balanced networks" show irregular and asynchronous spiking dynamics through a complex, sometimes chaotic, network state [6]. Nevertheless, the statistics of balanced networks are amenable to mean-field analysis [7-11], using techniques developed for spin-glass systems [12]. This analysis has given important insights into the circuit requirements that networks must satisfy to capture cortical-like dynamics. Subsequent experiments in cortex lend support to balanced network states with measurements of large and opposing excitatory and inhibitory synaptic currents [13,14], asynchronous cortical activity [15], as well as the sensitivity of network dynamics to small perturbations [16]. Further, balanced network models

Published by the American Physical Society under the terms of the Creative Commons Attribution 3.0 License. Further distribution of this work must maintain attribution to the author(s) and the published article's title, journal citation, and DOI.
Subject Areas: Biological Physics, Complex Systems

have made recent advances in theories of working memory [17], predictive neural coding [18], and stimulus representation [19].

Past work in balanced networks has assumed a spatially homogeneous $[6,9,11]$ or discretely clustered topology [20]. However, it is well established that the probability that two cortical neurons are connected depends on their separation in physical space or, for some sensory systems, feature space [21-24]. There has been substantial theoretical work on the spatiotemporal dynamics of phenomenological "neural-field-type" macroscale models of cortex [25]. However, treatments of neural variability in these frameworks have either assumed an external source of fluctuations [26-28] or that neurons are intrinsically Markovian [29,30]. In both cases, the stochastic aspects of the microscale system are imposed, in contrast to the internally generated variability in balanced networks. The capacity for pattern formation and spatial filtering in balanced networks with spatially dependent connection probabilities has not been addressed.

In this paper, we derive experimentally testable conditions on the strength and spatial profile of connection probabilities that must be satisfied for a recurrent network of excitatory and inhibitory neuron models to maintain a stable balanced state in the continuum limit. Specifically, we find that external inputs must be broader than recurrent excitation, which in turn must be broader than or equal to recurrent inhibition. Consequently, network models that rely on broad lateral inhibition to achieve sharpened tuning curves [31] or spatiotemporal dynamics [25,32] are inconsistent with the balanced state. Finally, we numerically investigate spatiotemporal spiking dynamics when stable balanced solutions do not exist. 


\section{NETWORK MODEL}

We consider a network of $N$ integrate-and-fire neurons, $N_{e}=q N$ of which are excitatory and $N_{i}=(1-q) N$ inhibitory, spaced evenly on the state space $\Gamma=(0,1]$, so that the $k$ th excitatory neuron is at location $x=k / N_{e}$ and similarly for the $k$ th inhibitory neuron. The input current to the $k$ th excitatory $(\alpha=e)$ and inhibitory $(\alpha=i)$ neuron is given by

$$
I_{\alpha}(x, t)=\sum_{j=1}^{N_{e}} J_{\alpha e}^{k, j} s_{e, j}(t)-\sum_{j=1}^{N_{i}} J_{\alpha i}^{k, j} s_{i, j}(t)+J_{\alpha}(x),
$$

respectively, where $x=k / N_{\alpha}$ and $s_{e, j}(t)=\sum_{i} \delta\left(t-t_{e, j}^{i}\right)$ is the spike train of the $j$ th excitatory neuron and similarly for $s_{i, j}(t)$. Static external input is provided by the terms $J_{\alpha}(x)$. The synaptic weight $J_{\alpha \beta}^{k j}$ is equal to the constant $J_{\alpha \beta}$ with probability $k_{\alpha \beta}^{\Gamma}(x-y)$, else it is zero. Here, $x=k / N_{\alpha}$, $y=j / N_{\beta}$, and

$$
k_{\alpha \beta}^{\Gamma}(x)=\sum_{n=-\infty}^{\infty} k_{\alpha \beta}(x+n)
$$

is the periodic summation of $k_{\alpha \beta}(x)$ for $\alpha, \beta \in\{e, i\}$. Thus, $\Gamma$ has periodic boundaries and $k_{\alpha \beta}$ is the spatial profile of $\beta$ to $\alpha$ connectivity. See Fig. 1 for a diagram of the network. As in Refs. [6,7], we fix $k_{\alpha \beta}(x) \ll 1$ to assure weak

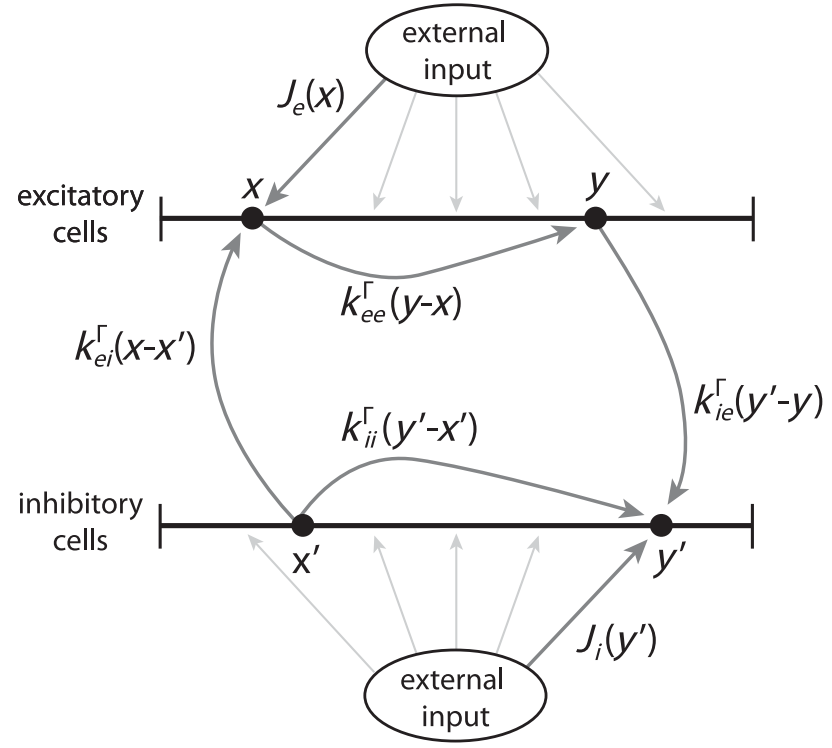

FIG. 1. Network diagram. Excitatory and inhibitory cells are arranged on the one-dimensional state space $\Gamma=(0,1]$ with periodic boundary conditions. An excitatory cell at location $x$ receives input from an inhibitory cell at location $x^{\prime}$ with probability $k_{e i}^{\Gamma}\left(x-x^{\prime}\right)$, and similarly for $k_{e e}^{\Gamma}, k_{i e}^{\Gamma}$, and $k_{i i}^{\Gamma}$, where $k_{\alpha \beta}^{\Gamma}(x)$ is the periodic summation of $k_{\alpha \beta}(x)$ on $\Gamma$; see Eq. (2). An excitatory cell at location $x$ receives temporally constant external input with intensity $J_{e}(x)$, and similarly for $J_{i}(x)$. correlations and we then consider the behavior of the network as $N \rightarrow \infty$.

Cortical neurons receive a large number of highamplitude excitatory inputs, implying that a postsynaptic cell requires only a fraction of excitatory presynaptic cells to drive spike responses [33]. Following past studies in balanced networks $[6,7,11]$, we model this with an $O(1)$ distance between rest and spike threshold and consider $J_{\alpha \beta} \sim O(1 / \sqrt{N}), \quad q \sim O(1), \quad k_{\alpha \beta} \sim O(1), \quad$ and $J_{\alpha}(x) \sim$ $O(\sqrt{N})$ as $N \rightarrow \infty$. To simplify calculations, we define $j_{\alpha \beta}=J_{\alpha \beta} \sqrt{N}, j_{\alpha}(x)=J_{\alpha}(x) / \sqrt{N}$, which do not depend on $N$. Note that scaling parameters in terms of the network size $N$ are equivalent to the common practice of scaling parameters in terms of the average number of inputs to each cell (often denoted as $K$ ) since the number of inputs to a cell scales linearly with $N$.

Under these scaling assumptions, a neuron receives recurrent input from $O(N)$ excitatory neurons but requires only $O(\sqrt{N})$ excitatory inputs to be active in an integration window to produce a spike. Finite, unsaturated firing rates can, therefore, be maintained only in the continuum limit through a dynamically stable balance between excitation and inhibition $[6,7,11]$. We next derive conditions under which such a stable balanced network state exists.

\section{CONDITIONS ON THE EXISTENCE OF A BALANCED STATE IN THE CONTINUUM LIMIT}

The mean firing rates of neurons in the network are denoted by $\nu_{\alpha}(x)=\left\langle E\left[s_{\alpha, k}(t)\right]\right\rangle$, where $x=k / N_{\alpha}, E[\cdot]$ represents expectation over realizations of network connectivity and $\langle\cdot\rangle$ the average over time. In the continuum limit, the mean input currents are related to the firing rates by

$$
\begin{aligned}
\mu_{\alpha}(x) & :=\left\langle E\left[I_{\alpha}(x, t)\right]\right\rangle \\
& =\sqrt{N}\left[w_{\alpha e} * \nu_{e}(x)-w_{\alpha i} * \nu_{i}(x)+j_{\alpha}(x)\right],
\end{aligned}
$$

for $\alpha=e, \quad i$, where $w_{\alpha e}(x)=q j_{\alpha e} k_{\alpha e}^{\Gamma}(x), \quad w_{\alpha i}(x)=$ $(1-q) j_{\alpha i} k_{\alpha i}^{\Gamma}(x)$, and $*$ denotes circular convolution on $\Gamma$. We aim to derive conditions under which $\nu_{\alpha}(x)$ and $\mu_{\alpha}(x)$ each converge to a finite limit as $N \rightarrow \infty$ and $\nu_{\alpha}(x)$ does not become identically zero. For these conditions to be realized, we must have that

$$
w_{\alpha e} * \nu_{e}(x)-w_{\alpha i} * \nu_{i}(x)+j_{\alpha}(x)=O(1 / \sqrt{N}) .
$$

Taking $N \rightarrow \infty$ gives a Fredholm equation of the first kind, which can be written in the Fourier domain as

$$
\tilde{w}_{e e} \tilde{\nu}_{e}-\tilde{w}_{e i} \tilde{\nu}_{i}+\tilde{j}_{e}=0, \tilde{w}_{i e} \tilde{\nu}_{e}-\tilde{w}_{i i} \tilde{\nu}_{i}+\tilde{j}_{i}=0,
$$

with the solution given in the Fourier domain by 


$$
\tilde{\nu}_{e}=\frac{\tilde{j}_{e} \tilde{w}_{i i}-\tilde{j}_{i} \tilde{w}_{e i}}{\tilde{w}_{e i} \tilde{w}_{i e}-\tilde{w}_{e e} \tilde{w}_{i i}}, \quad \tilde{\nu}_{i}=\frac{\tilde{j}_{e} \tilde{w}_{i e}-\tilde{j}_{i} \tilde{w}_{e e}}{\tilde{w}_{e i} \tilde{w}_{i e}-\tilde{w}_{e e} \tilde{w}_{i i}},
$$

where $\tilde{f}(n)=\int_{\Gamma} e^{-2 \pi x n i} f^{\Gamma}(x) d x$. Equation (5) must hold at every Fourier mode $n$ for which

$$
\tilde{w}_{e i}(n) \tilde{w}_{i e}(n)-\tilde{w}_{e e}(n) \tilde{w}_{i i}(n) \neq 0 .
$$

If $\tilde{w}_{e i}(n) \tilde{w}_{i e}(n)-\tilde{w}_{e e}(n) \tilde{w}_{i i}(n)=0$ at some Fourier mode, then for a solution to exist, it must also be true that

$\tilde{j}_{e}(n) \tilde{w}_{i i}(n)-\tilde{j}_{i}(n) \tilde{w}_{e i}(n)=\tilde{j}_{e}(n) \tilde{w}_{i e}(n)-\tilde{j}_{i}(n) \tilde{w}_{e e}(n)=0$

at that Fourier mode.

Requiring firing rates from Eq. (5) to be non-negative and not identically zero implies that

$$
\frac{\bar{j}_{e}}{\bar{j}_{i}}>\frac{\bar{w}_{e i}}{\bar{w}_{i i}}>\frac{\bar{w}_{e e}}{\bar{w}_{i e}}
$$

or

$$
\frac{\bar{j}_{e}}{\bar{j}_{i}}<\frac{\bar{w}_{e i}}{\bar{w}_{i i}}<\frac{\bar{w}_{e e}}{\bar{w}_{i e}},
$$

where $\bar{f}=\tilde{f}(0)=\int_{\Gamma} f^{\Gamma}(x) d x$ is the average value of $f$ on $[0,1]$. Note that Eq. (6) is equivalent to a balance condition derived in Ref. [7] for spatially homogeneous networks. We show below that Eq. (6) leads to a stable balanced state for large $N$ but Eq. (7) does not.

The solutions in Eq. (5) are viable only if $\tilde{\nu}_{\alpha}$ has a welldefined inverse Fourier transform, which requires at least that

$$
\lim _{n \rightarrow \infty} \frac{\tilde{j}_{e}(n) \tilde{w}_{i \alpha}(n)-\tilde{j}_{i}(n) \tilde{w}_{e \alpha}(n)}{\tilde{w}_{e i}(n) \tilde{w}_{i e}(n)-\tilde{w}_{e e}(n) \tilde{w}_{i i}(n)}=0,
$$

for $\alpha=e, i$. This condition effectively states that external inputs must be broader than recurrent connections, since their Fourier coefficients must decay more slowly. To understand why this must be true, consider Eq. (4) when, for example, all $w_{\alpha \beta}(x)$ terms are broader than all $j_{\alpha}(x)$ terms. Since convolution can only broaden functions, all $w_{\alpha \beta} * \nu_{\beta}(x)$ terms are necessarily broader than all $j_{\alpha}(x)$ for any choice of $\nu_{\alpha}(x)$ and, therefore, a solution to Eq. (4) cannot be obtained. We investigate this condition for Gaussian-shaped inputs and connectivity kernels next.

\section{EXAMPLE WITH GAUSSIAN-SHAPED CONNECTIVITY}

For ease of exposition, we consider connectivity kernels and inputs that have Gaussian shape, and we assume that the probability (but not the strength) of a connection depends only on presynaptic cell type. Specifically, we take

$$
w_{\alpha \beta}(x)=\bar{w}_{\alpha \beta} g\left(x ; 0, \sigma_{\beta}\right)
$$

and

$$
j_{\alpha}(x)=p \bar{j}_{\alpha} g\left(x ; x_{o}, \sigma_{o}\right)+(1-p) \bar{j}_{\alpha} .
$$

Here, $\bar{w}_{\alpha \beta}$ and $\bar{j}_{\alpha}$ are constants and

$$
g(x ; \mu, \sigma)=\frac{1}{\sqrt{2 \pi} \sigma} \sum_{n=-\infty}^{\infty} e^{-(x+n-\mu)^{2} / 2 \sigma^{2}}
$$

is a wrapped Gaussian for $x \in[0,1]$. In the Fourier domain, the wrapped Gaussian is given by

$$
\tilde{g}(n ; \mu, \sigma)=e^{-2 n^{2} \pi^{2} \sigma^{2}-2 n \pi \mu i} .
$$

In this case, the balance condition in Eq. (8) is satisfied only if $\sigma_{o}>\sigma_{e}, \sigma_{i}$. Hence, external inputs must be spatially broader than recurrent connections for a balanced solution to exist. Under this condition, taking the inverse transform in Eq. (5) gives the balanced solution

$$
\nu_{\alpha}(x)=p \bar{\nu}_{\alpha} g\left(x ; x_{o}, \sqrt{\sigma_{o}^{2}-\sigma_{\alpha}^{2}}\right)+(1-p) \bar{\nu}_{\alpha},
$$

where $\bar{\nu}_{\alpha}=\tilde{\nu}_{\alpha}(0)$ from Eq. (5). Note that the peaked shape of the firing rate profile in Eq. (9) is inherited by the peaked shape of the inputs, while the width of the firing rate profiles depends on the difference of the input width $\left(\sigma_{o}^{2}\right)$ and the recurrent spatial filtering $\left(\sigma_{\alpha}^{2}\right)$. Flat inputs $(p=0)$ lead to a flat firing rate profile $\left[\nu_{\alpha}(x)=\bar{\nu}_{\alpha}\right]$.

The analysis of the balanced state above is valid in the $N \rightarrow \infty$ limit for a large class of neuron models [9]. To numerically validate our theoretical results, we use a leaky integrate-and-fire (LIF) model in which the membrane potential of a cell in population $\alpha$ at location $x$ satisfies

$$
V^{\prime}(t)=-\frac{1}{\tau_{m}} V(t)+I_{\alpha}(x, t),
$$

with a reflecting barrier at $V=-1$, where $\tau_{m}=20 \mathrm{~ms}$. Spikes occur whenever $V=1$, at which point $V(t)$ is reset to zero. Steady-state firing rates can be approximated numerically using Monte Carlo simulations of the full LIF network or semianalytically by locating fixed points of a diffusion approximation. The diffusion approximation is given by replacing $I_{\alpha}(x, t)$ in Eq. (10) by $\mu_{\alpha}(x)+$ $\sqrt{2 D_{\alpha}(x)} \eta_{\alpha}(x, t)$, where each $\eta_{\alpha}(x, t)$ is an independent realization of Gaussian white noise and [8]

$$
\begin{aligned}
D_{\alpha}(x) & \approx \frac{1}{2} \lim _{\delta \rightarrow 0} \delta^{-1}\left\langle E\left[\int_{t}^{t+\delta} I_{\alpha}(x, s)-\mu_{\alpha}(x) d s\right]^{2}\right\rangle \\
& =\frac{j_{\alpha e}}{2} w_{\alpha e} * \nu_{e}(x)+\frac{j_{\alpha i}}{2} w_{\alpha i} * \nu_{i}(x)
\end{aligned}
$$


is the temporal diffusion coefficient. The spatial independence of $\eta$ is reasonable because the sparse coupling in the network ensures that common input to neuron pairs is negligible.

Under the diffusion approximation, steady-state firing rates are obtained by numerically searching for a fixed point $\left[\nu_{e}^{0}(x), \nu_{i}^{0}(x)\right]$ that satisfies

$$
\nu_{\alpha}^{0}(x)=\phi\left(\mu_{\alpha}^{0}(x), D_{\alpha}^{0}(x)\right) .
$$

Here, $\phi(\mu, D)$ relates input mean and variance to firing rate of the LIF model for the diffusion approximation and where $\mu_{\alpha}^{0}(x)$ and $D_{\alpha}^{0}(x)$ are given in terms of $\nu_{e}^{0}(x)$ and $\nu_{i}^{0}(x)$ by Eqs. (3) and (11). The function $\phi(\mu, D)$ is known in closed form [34], but is more efficiently calculated by solving a boundary value problem [35]. Numerical solutions to the fixed point problem in Eq. (12) were used for the curves labeled "FP" in Figs. 3 and 4. The default parameter values for all simulations are $\sigma_{e}=\sigma_{i}=0.1, \sigma_{o}=0.2, j_{e e}=0.5$,
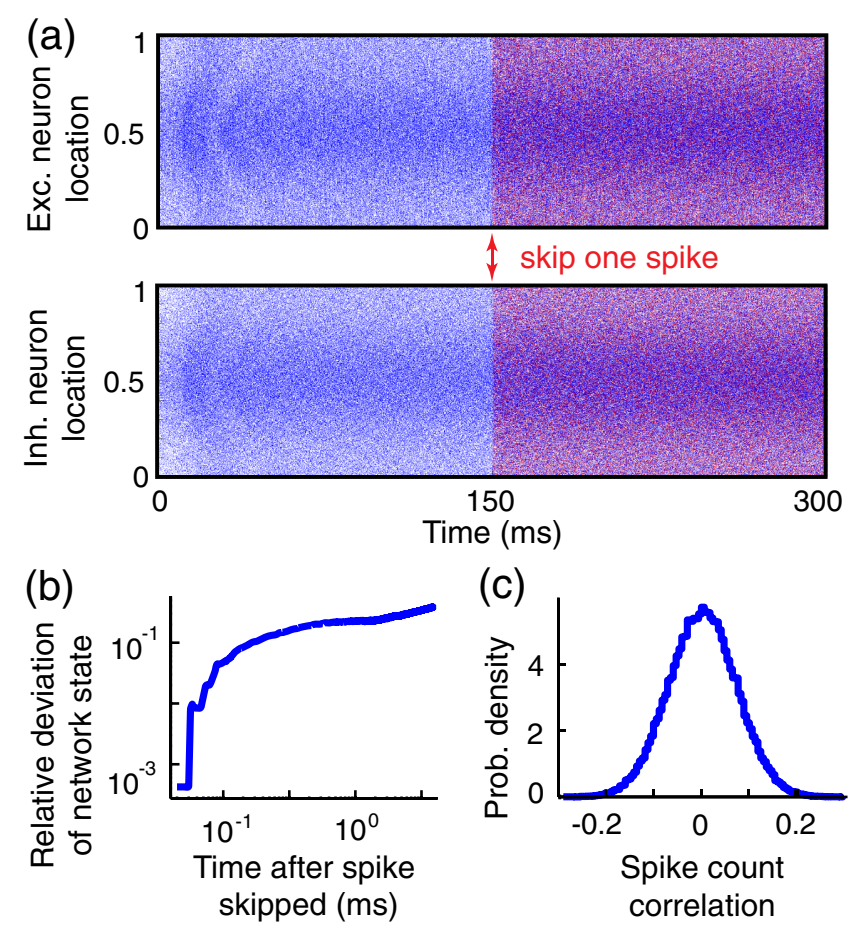

FIG. 2. Balanced network dynamics. (a) Raster plots from two Monte Carlo simulations with identical initial states (blue and red dots, respectively). In one simulation (red dots), the first spike after $150 \mathrm{~ms}$ is skipped, revealing a sensitivity to perturbations. (b) The normalized $L^{2}$ deviation between the vector of membrane potentials of the two network simulations from (a) as a function of time elapsed since a spike was skipped. (c) Histogram of spike count correlations between neighboring neurons (mean of $4.84 \times 10^{-4}$ and standard deviation of 0.0711 ). Correlations computed by counting spikes over a $300 \mathrm{~ms}$ window in each neuron from 200 simulations of the same network realization with different initial conditions. All panels from network simulations with $N=10^{5}$ neurons. $j_{e i}=1, \quad j_{i e}=0.7, \quad j_{i i}=1, \quad \bar{j}_{e}=4 \times 10^{-4}(\mathrm{~ms})^{-1}$, $\bar{j}_{i}=3 \times 10^{-4}(\mathrm{~ms})^{-1}, q=0.5, p=0.25$, and $\bar{k}_{\alpha \beta}=0.02$ for $\alpha, \beta \in\{e, i\}$. Any deviations from these parameters are explicitly denoted in the figure captions and legends.

When the balanced state exists, simulations of the LIF network show asynchronous and irregular spiking dynamics [Fig. 2(a)]. The microscopic state of the network is highly sensitive to the deletion of a single spike [Figs. 2(a) and 2(b)], but sufficiently small perturbations of the membrane potentials do not cause a divergence of trajectories (not pictured). These findings are consistent with previous studies showing that balanced networks can exhibit "stable chaos" characterized by exponentially long transients, sensitivity to small but finite perturbations and insensitivity to sufficiently small perturbations [10,36-39]. The distribution of Pearson correlation coefficients between the spike counts of neighboring neurons is approximately Gaussian shaped with a mean near zero despite the fact that neighboring neurons share more than $5 \%$ of their inputs on average [Fig. 2(c)], consistent with the network having reached a stable asynchronous state [11].

Despite the sensitivity of the microscopic dynamics, the macroscopic dynamics, measured by the network firing rates, are stable to the deletion of spikes. The neuron firing rates are given by the fixed point of Eq. (12), which converges to the balanced fixed point given by Eq. (9) as the network size increases (Fig. 3). Thus, our mean-field analysis gives an accurate description of the spatial
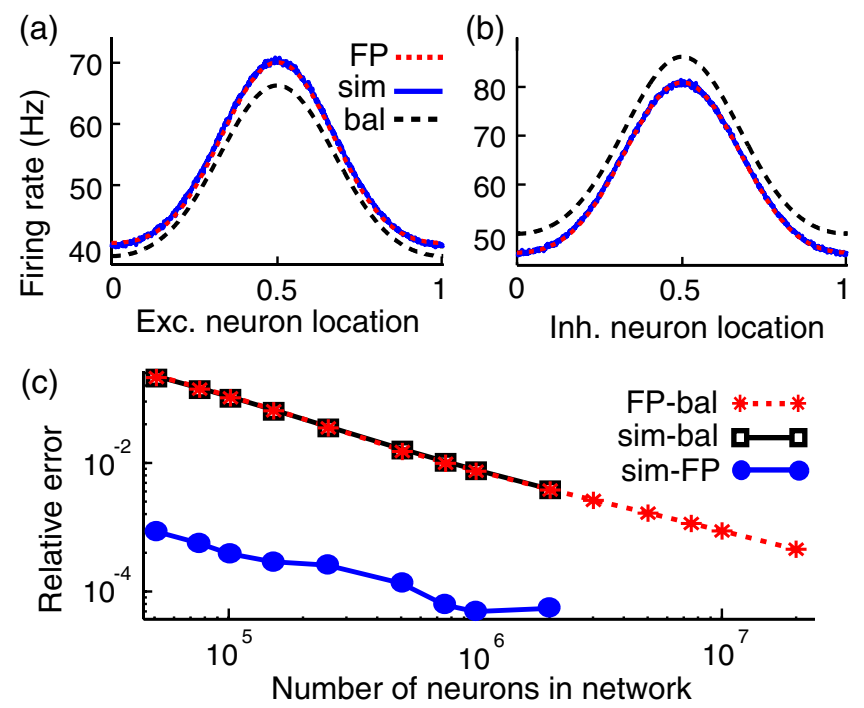

FIG. 3. Balanced network firing rates. (a) Population firing rates $\nu_{e}(x)$ and $\nu_{i}(x)$ calculated from full network simulations ("sim," solid blue line, $N=2 \times 10^{6}$ ), the numerically computed fixed point in Eq. (12) ("FP,' dotted red line, $N=2 \times 10^{6}$ ), and the balanced solution from Eq. (9) ("bal," dashed black line). (b) Relative meansquare difference between the firing rates calculated using the three methods from (a) at various values of $N$. 
distribution of population activity for sufficiently large networks.

\section{SPATIALLY IMBALANCED NETWORKS}

An $O(c)$ deviation of the firing rates away from balance yields an $O(c \sqrt{N})$ deviation of the mean input currents, but only an $O(c)$ perturbation of the input variance, cf. Eqs. (3-11). When mean input is large in magnitude, the firing rate transfer of a LIF neuron can be approximated as threshold linear, motivating the following mean-field approximation to firing rate dynamics:

$$
\tau \frac{\partial \nu_{\alpha}}{\partial t}=-\nu_{\alpha}+\gamma \mu_{\alpha} \Theta\left(\mu_{\alpha}\right)
$$

Here, $\Theta(\cdot)$ is the Heaviside function, $\tau$ is the characteristic time scale of the network, $\gamma>0$ is the gain of the neuron, and $\mu_{\alpha}$ is related to $\nu_{\beta}$ through Eq. (3) for $\alpha, \beta \in\{e, i\}$. For all figures, we use $\gamma=1$, which is valid for the LIF model described above when mean input is large. Equation (13) can be solved for finite $N$ and will provide intuition for network solutions when the condition in Eq. (8) is violated.

If Eq. (13) admits a fixed point with strictly positive firing rates, it is given in the Fourier domain by

$$
\begin{aligned}
& \tilde{\nu}_{e}^{0}=\frac{\epsilon \tilde{j}_{e}+\tilde{j}_{e} \tilde{w}_{i i}-\tilde{j}_{i} \tilde{w}_{e i}}{\epsilon^{2}-\epsilon \tilde{w}_{e e}+\epsilon \tilde{w}_{i i}+\tilde{w}_{e i} \tilde{w}_{i e}-\tilde{w}_{e e} \tilde{w}_{i i}}, \\
& \tilde{\nu}_{i}^{0}=\frac{\epsilon \tilde{j}_{i}+\tilde{j}_{e} \tilde{w}_{i e}-\tilde{j}_{i} \tilde{w}_{e e}}{\epsilon^{2}-\epsilon \tilde{w}_{e e}+\epsilon \tilde{w}_{i i}+\tilde{w}_{e i} \tilde{w}_{i e}-\tilde{w}_{e e} \tilde{w}_{i i}},
\end{aligned}
$$

(a)

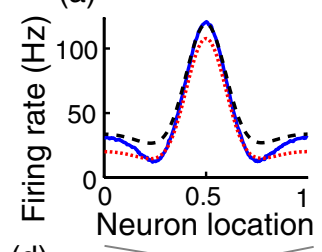

(d)

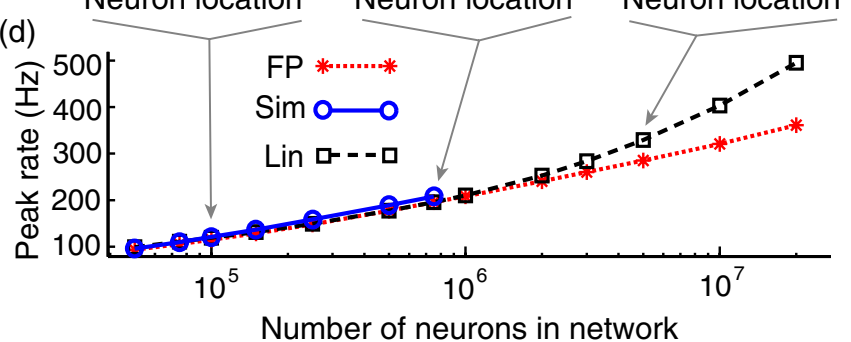

FIG. 4. Loss of balanced state when external inputs are narrower than recurrent connections. Firing rates of the excitatory population when external inputs are narrower than recurrent inputs for system sizes (a) $N=10^{5}$, (b) $N=7.5 \times 10^{5}$, and (c) $N=5 \times 10^{6}$. (d) Peak firing rate of the excitatory population as a function of system size. In all panels, $\sigma_{o}=0.1$, $\sigma_{e}=\sigma_{i}=0.2$, and other parameters are as in Fig. 3. Dotted red curves computed by numerically solving Eq. (12). Solid blue curves computed from full network simulations. Dashed black line computed from the linear approximation given in Eq. (14).

where $\epsilon=1 /(\gamma \sqrt{N})$. If Eq. (8) is satisfied $\left(\sigma_{o}>\sigma_{e}, \sigma_{i}\right)$, then the fixed point in Eq. (14) converges to the balanced solution in Eq. (5) as $N \rightarrow \infty$. If Eq. (8) is violated $\left(\sigma_{o} \leq \sigma_{e}, \sigma_{i}\right)$, then the higher spatial Fourier modes, and therefore peak firing rates, from Eq. (14) diverge as $N \rightarrow \infty$ (Fig. 4). Eventually, this growth of higher Fourier modes causes $\nu_{\alpha}(x)<0$ for some $x$ [Fig. 4(c)], at which point Eq. (14) no longer reflects a fixed-point solution to Eq. (13). Regardless, peak firing rates continue to grow as $N$ increases when $\sigma_{o}<\sigma_{e}, \sigma_{i}$ [Fig. 4(d)].

\section{STABILITY OF THE BALANCED STATE}

The balanced fixed point from Eq. (14) is stable for the mean-field model in Eq. (13) whenever

$$
A(n)=\left[\begin{array}{cc}
-\epsilon+\tilde{w}_{e e}(n) & -\tilde{w}_{e i}(n) \\
\tilde{w}_{i e}(n) & -\epsilon-\tilde{w}_{i i}(n)
\end{array}\right]
$$

has eigenvalues with negative real part or, equivalently, when

$$
\tilde{w}_{e i} \tilde{w}_{i e}-\tilde{w}_{e e} \tilde{w}_{i i}>\epsilon\left(\tilde{w}_{e e}-\tilde{w}_{i i}\right)-\epsilon^{2} \text { and } \tilde{w}_{e e}-\tilde{w}_{i i}<2 \epsilon
$$

at each Fourier mode $n$. For the Gaussian-shaped kernels described above, stability of the balanced state as $N \rightarrow \infty$ $(\epsilon \rightarrow 0)$ under this approximation requires that $\bar{w}_{e e}<\bar{w}_{i i}$

(a)

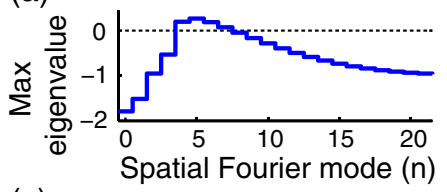

(c)
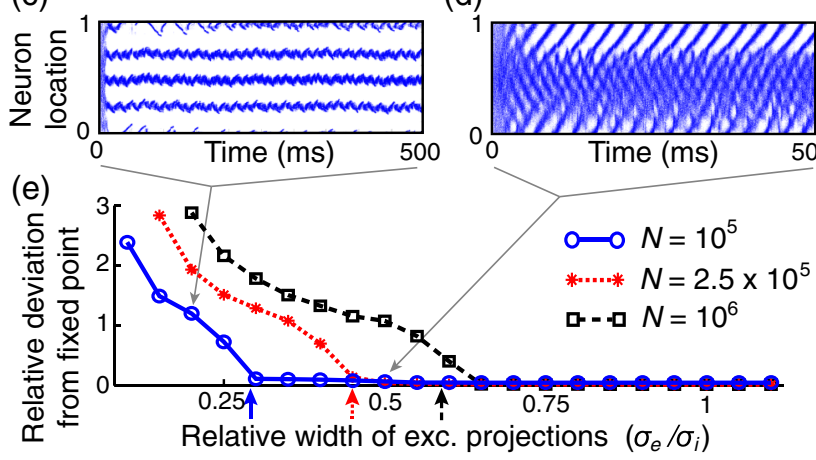

FIG. 5. Instability of the balanced state when recurrent excitation is too narrow compared to inhibition. (a),(b) Maximum real part of eigenvalues of the matrix $A(n)$ from Eq. (15) as a function of $n$ with $\sigma_{e}=0.02$ in (a) and $\sigma_{e}=0.05$ in (b) (other parameters as in Fig. 2). (c),(d) Spike rasters from simulations of the LIF network. (e) Relative $L^{2}$ deviation of simulated firing rates from the fixed point determined by Eq. (12) for various values of $\sigma_{e}$ and $N$ (see inset). Vertical arrows along the horizontal axis mark the smallest value of $\sigma_{e} / \sigma_{i}$ at which some eigenvalue of $A(n)$ has positive real part. 
and $\sigma_{e} \geq \sigma_{i}$ are satisfied in addition to Eq. (6), but networks satisfying Eq. (7) do not satisfy Eq. (16) for large $N$. The mean-field model predicts instabilities of the balanced state for full network simulations reasonably well (Fig. 5). In particular, when $\sigma_{e}$ is sufficiently smaller than $\sigma_{i}, A(n)$ has eigenvalues with positive real part, the balanced fixed point loses stability, and different spatial patterns are produced [Figs. 5(a), 5(c), and 5(e)].

For $\sigma_{e} / \sigma_{i}$ near the stability transition, the network exhibits waves of activity, but the time-averaged firing rates remain close to the balanced fixed point [Figs. 5(b), 5(d), and 5(e)]. The direction that these waves travel depends on initial conditions even when the network architecture remains fixed (data not shown), suggesting a symmetry-breaking multistability. This spatiotemporally coherent activity is not captured by the mean-field model in Eq. (13), even when the threshold-linear rate function is replaced by the actual nonlinear function $\phi(\mu, D)$. A theoretical description of these patterns is outside the scope of this study. A complete stability analysis could potentially be achieved using methods from Ref. [40]. Regardless, our analysis of the mean-field approximation provides a useful explanation for why the balanced state becomes unstable when excitatory projections are too much narrower than inhibitory projections.

\section{OTHER SPATIAL TOPOLOGIES}

Thus far, we have considered a one-dimensional model with periodic boundary conditions for simplicity, but our analysis can be adapted to different spatial topologies.

\section{A. Two-dimensional networks}

The one-dimensional network is easily extended to a twodimensions state space $\Gamma=(0,1] \times(0,1]$, where neurons are arranged uniformly on a lattice. In this network, a neuron in population $\alpha$ at location $\left(x_{1}, y_{1}\right)$ receives input from a neuron in population $\beta$ at location $\left(x_{2}, y_{2}\right)$ with probability $k_{\alpha \beta}^{\Gamma}\left(x_{2}-x_{1}, y_{2}-y_{1}\right)$, where $k^{\Gamma}(x, y)=\sum_{m, n=-\infty}^{\infty} k(x+$ $m, y+n)$ creates periodic boundaries and $\alpha, \beta \in\{e, i\}$. Given two-dimensional inputs $J_{\alpha}(x, y)$ and defining $j_{\alpha}(x, y)$, $j_{\alpha \beta}$, and $w_{\alpha \beta}(x, y)$ analogously to the one-dimensional case, the balanced firing rate solutions are given by Eq. (5), where $\tilde{f}(m, n)=\int_{0}^{1} \int_{0}^{1} e^{-2 \pi i(x n+y m)} f(x, y) d x d y$ is a twodimensional Fourier series. The existence conditions in Eqs. (6) and (7) are identical in two dimensions with $\bar{f}=\tilde{f}(0,0)$. The existence condition in Eq. (8) is identical except the limit must be taken in $m$ and $n$ simultaneously. The stability conditions in Eq. (16) are also identical in two dimensions, but need to be satisfied at each Fourier mode pair $(m, n)$.

The example with Gaussian-shaped inputs and connectivity kernels can be extended to two dimensions by defining

$$
w_{\alpha \beta}(x, y)=\bar{w}_{\alpha \beta} G\left(x, y ; 0,0, \Sigma_{\beta}\right)
$$

and

$$
j_{\alpha}(x, y)=p \bar{j}_{\alpha} G\left(x, y ; x_{o}, y_{o}, \Sigma_{o}\right)+(1-p) \bar{j}_{\alpha},
$$

where

$$
\begin{aligned}
& G\left(x, y ; \mu_{x}, \mu_{y}, \Sigma\right) \\
& \quad=\frac{1}{2 \pi \sqrt{\operatorname{det}(\Sigma)}} \sum_{m, n=-\infty}^{\infty} e^{-(1 / 2)\left[(\vec{x}-\vec{\mu}+\vec{n}) \Sigma^{-1}(\vec{x}-\vec{\mu}+\vec{n})^{T}\right]}
\end{aligned}
$$

is a two-dimensional wrapped Gaussian, $\vec{x}=[x y]$, $\vec{\mu}=\left[\mu_{x} \mu_{y}\right], \vec{n}=[m n]$, and $\Sigma$ is a $2 \times 2$ positive-definite matrix. The existence condition in Eq. (8) is satisfied whenever $\Sigma_{o}-\Sigma_{\alpha}$ is a positive-definite matrix for $\alpha=e, i$, generalizing the $\sigma_{o}>\sigma_{\alpha}$ condition from the one-dimensional case. Under this condition, the balanced solutions are given by

$$
\nu_{\alpha}(x, y)=p \bar{\nu}_{\alpha} G\left(x, y ; x_{o}, y_{o}, \Sigma_{o}-\Sigma_{\alpha}\right)+(1-p) \bar{\nu}_{\alpha},
$$

for $\alpha=e, i$, where $\bar{\nu}_{\alpha}=\tilde{\nu}_{\alpha}(0,0)$ is given by Eq. (5). These solutions are stable as $N \rightarrow \infty$ whenever Eq. (6) is satisfied, $\bar{w}_{e e}>\bar{w}_{i i}$ and $\Sigma_{e}-\Sigma_{i}$ is positive definite. If we assume that
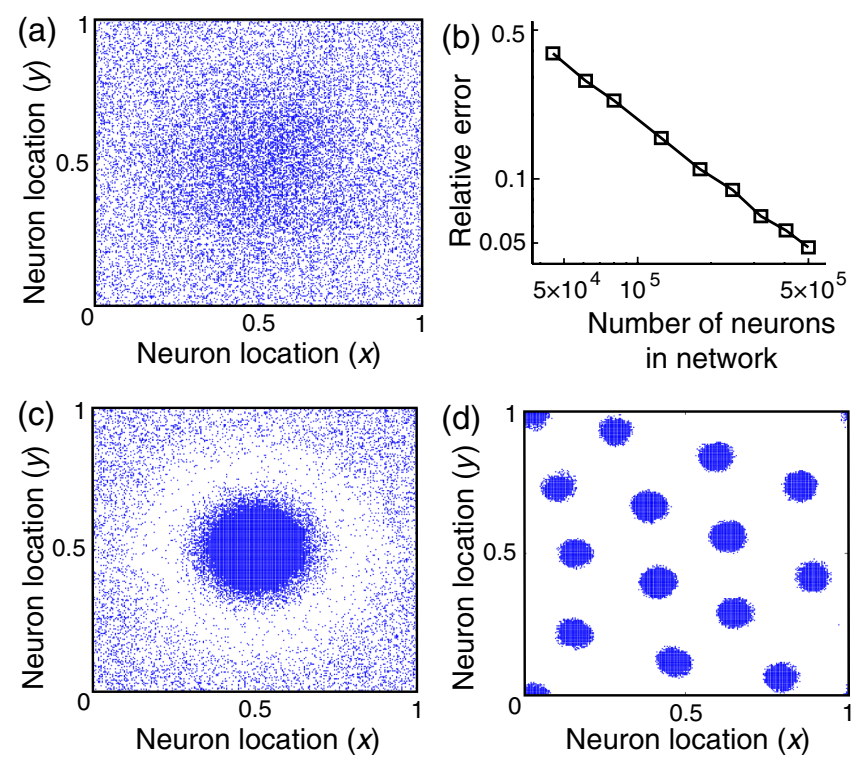

VIDEO 1. Two-dimensional network simulations. (a) Raster plot of excitatory neuron spikes from a two-dimensional network simulation with $\sigma_{o}=0.2$ and $\sigma_{e}=\sigma_{i}=0.1$, so that a stable balanced state exists. Excitatory and inhibitory neurons are each arranged on a uniform $250 \times 250$ lattice, for a total of $N=$ $1.25 \times 10^{5}$ neurons. (b) The relative mean-square difference between the firing rates calculated from the simulation in (a) and from the balanced fixed point in Eq. (17) (log-log scale). (c) Same as (a) except $\sigma_{o}=0.1$ and $\sigma_{e}=\sigma_{i}=0.2$, so that a balanced fixed point does not exist. (d) Same as (a) except $\sigma_{e}=0.02$. Open video file in a web browser to view animation. 
the network structure is isotropic so that $\Sigma_{e}=\sigma_{e}^{2} I$, $\Sigma_{i}=\sigma_{i}^{2} I$, and $\Sigma_{o}=\sigma_{o}^{2} I$, where $I$ is the $2 \times 2$ identity matrix, then existence and stability of the balanced state requires that $\sigma_{o}>\sigma_{e} \geq \sigma_{i}$, in analogy to the onedimensional network.

We simulated a two-dimensional isotropic network with the same parameters used for the one-dimensional simulations $\left(\sigma_{o}=0.2, \sigma_{e}=\sigma_{i}=0.1\right)$. Spiking dynamics were qualitatively similar to the one-dimensional network [Video 1(a)] and firing rates converged toward the balance fixed point given in Eq. (17) as $N$ increased [Video 1(b)]. Changing parameters so that the external input is narrower than the recurrent projections $\left(\sigma_{o}=0.1, \sigma_{e}=\sigma_{i}=0.2\right)$ prevents the existence of a balanced fixed point and induces a surround-suppression pattern with extremely high peak firing rates [Video 1(c), peak excitatory rate over $500 \mathrm{~Hz}$ ] in analogy to one-dimensional simulations (compare to Fig. 4). Sharpening the profile of recurrent excitation $\left(\sigma_{o}=0.2, \sigma_{e}=0.02, \sigma_{i}=0.1\right)$ destabilizes the balanced state and induces spatial patterns [Video 1(d)] analogous to those observed in one-dimensional simulations [compare to Fig. 5(c)].

\section{B. Absorbing boundaries}

Our analysis of one- and two-dimensional networks relies on the assumption that connection probability obeys periodic boundary conditions, since this allows us to write Eq. (4) in terms of circular convolutions, which in turn allows Fourier modes to decouple. To test the sensitivity of network dynamics to the assumption of periodic boundary conditions, we simulate the one-dimensional network without periodic boundary conditions. Specifically, the
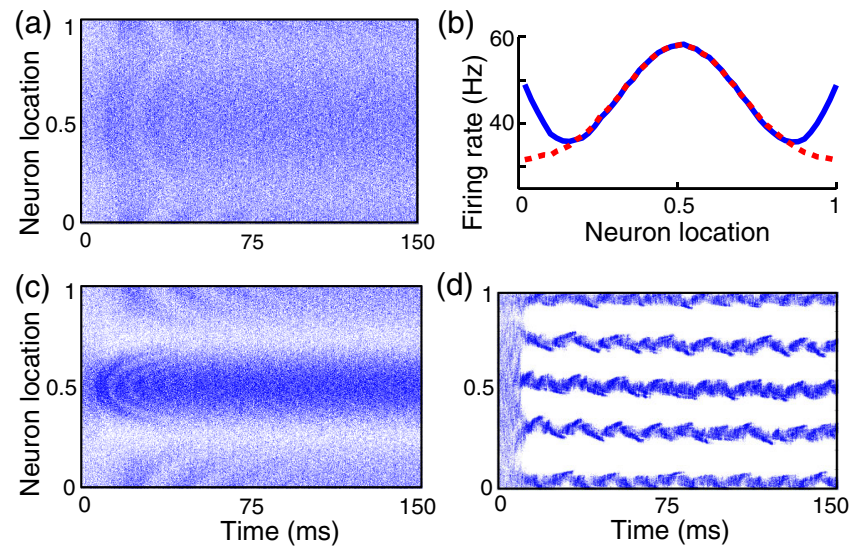

FIG. 6. Network simulations without periodic boundaries. (a) Raster plot of excitatory spike trains from a simulation identical to the simulation in Fig. 2(a) except that connection probability is computed without periodic boundaries (so that cells near the boundaries receive fewer recurrent inputs). (b) Firing rate profile of excitatory cells from (a) (solid blue curve) is similar to the firing rate profile from Fig. 2(a) (dashed red curve) except near the boundaries. (c) Same as (a) except $\sigma_{o}=0.1$ and $\sigma_{e}=\sigma_{i}=0.2$. (d) Same as (a) except $\sigma_{e}=0.02$. probability that $J_{\alpha \beta}^{k, j} \neq 0$ was set to $k_{\alpha \beta}(x-y)$ instead of $k_{\alpha \beta}^{\Gamma}(x-y)$. Hence, neurons near the boundaries receive less recurrent input.

Although this framework is more difficult to analyze, network simulations are qualitatively similar to simulations with periodic boundaries. When $\sigma_{o}>\sigma_{e}=\sigma_{i}$, spiking dynamics are similar to those with periodic boundaries [see Fig. 6(a) and compare to Fig. 2(a)] and firing rates differ only near the boundary [Fig. 6(b)]. When $\sigma_{o}<\sigma_{e}=\sigma_{i}$, a surround-suppression dynamic appears, with large peak firing rates [Fig. 6(c), peak excitatory rate $120 \mathrm{~Hz}$ ], similar to simulations with periodic boundaries (compare to Fig. 4). When $\sigma_{e}$ is sufficiently smaller than $\sigma_{i}$, a stable spatial pattern emerges [Fig. 6(d)], similar to those observed in one-dimensional simulations [compare to Fig. 5(c)].

\section{DISCUSSION}

By taking into account the spatial dependence of connection probabilities, we derive new conditions for the existence and stability of balanced solutions. With Gaussian connectivity, the conditions reduce to the inequality chain $\sigma_{o}>\sigma_{e} \geq \sigma_{i}$.

Consistent with this conclusion, several studies have found that thalamocortical projections are generally broader than intracortical projections [41-43] $\left(\sigma_{o}>\sigma_{e}\right)$ and circuit measurements in cortical layer 4 show that excitation projects more broadly than inhibition [24] $\left(\sigma_{e} \geq \sigma_{i}\right)$. In contrast, many previous models rely on broad inhibition to sharpen tuning curves $[44,45]$ and promote pattern formation $[25,32]$. Our results refute the notion that dynamical mechanisms relying on such broad inhibition can coexist with a balanced state. Nevertheless, Eq. (9) reveals that recurrent connections in our model sharpen tuning curves even when $\sigma_{e} \geq \sigma_{i}$ since the width of the firing rate profiles is the width of the external inputs minus the width of recurrent projections.

Our analysis relies on the assumption that connection probability depends only on distance and that boundaries are periodic. Similar analysis can be performed in an unbounded state space (i.e., all of $\mathbb{R}$ or $\mathbb{R}^{2}$ ). In this case, the analysis of the balanced state is identical to that for periodic boundaries except that a continuous Fourier spectrum is used in place of a discrete spectrum. The Gaussian examples and the conditions derived for them are also identical, except that the wrapped Gaussian is replaced by a full Gaussian.

A general treatment of spatially heterogeneous balanced networks would allow the probability that a cell at location $x$ receives input from a cell at location $y$ to depend on $x$ and $y$ independently, instead of just their distance. In this case, Eq. (4) is replaced by

$$
\int_{\Gamma} w_{\alpha e}(x, y) \nu_{e}(y)-w_{\alpha i}(x, y) \nu_{i}(y) d y+j_{\alpha}(x)=0
$$


in the $N \rightarrow \infty$ limit, but this equation cannot generally be written in terms of convolutions and, therefore, cannot be solved using the Fourier methods used to solve Eq. (4). Several techniques have been developed for Fredholm equations like Eq. (18), so analytical solutions are potentially tractable for certain choices of the functions $w_{\alpha \beta}(x, y)$ and inputs $j_{\alpha}(x)$ [46,47]. Moreover, numerical techniques can be used even when analytical approaches fail [48].

We observe extremely weak average correlations between the spiking activity of neighboring neurons even when they share over 5\% of their recurrent inputs [see Fig. 2(c) and related discussion], suggesting that the network is in the asynchronous state. The existence of the asynchronous state in balanced networks has been analyzed only for homogeneous networks of binary neurons [11]. We hope to extend this analysis to spatially heterogeneous networks of integrate-and-fire neurons in future work.

The majority of balanced network studies assume a homogeneous topology in which connection probability and external drive depend solely on cell type (excitatory or inhibitory). In previous studies of heterogeneous balanced networks, the relative difference between the total synaptic drive to differently tuned or spatially separated neurons scales like $O(1 / \sqrt{N})$ even though the absolute difference is $O(1)[20,49-51]$. As a result, these models do not need to satisfy the spatial balance conditions derived here since all heterogeneity is absorbed into the $O(1 / \sqrt{N})$ term in Eq. (4). These models predict that the relative difference between the excitatory (or inhibitory) drive to differently tuned or spatially separated neurons is small compared to the relative difference between their firing rates. Our model predicts that the relative difference between two neurons' excitatory or inhibitory drive is of the same order as the relative difference between their firing rates. This distinction could be used to distinguish between the models based on experimental recordings.

Spatially extended stochastic neural field models are typically constructed by appending additive noise to a deterministic model [30,32], similar to the practice of augmenting reaction diffusion systems with additive or multiplicative noise [1]. Analysis of neural-field models driven by external stochastic forcing shows that the spatiotemporal structure of noise is a critical determinant of the ensuing stochastic dynamics [26-28]. However, in all cases these spatially distributed systems with external stochastic forcing show how pattern-forming systems filter noise. By contrast, in balanced networks the variability arises naturally through internal mechanisms $[6,7,9,20]$, so that assumptions about the structure of external stochastic forcing are not required. Indeed, the network simulations in this study are completely deterministic, so that all temporal variability is generated through chaotic network dynamics. Thus, balanced networks with spatial interactions offer an alternative framework to stochastically forced neural-field models, where complex internal dynamics is the source, as opposed to filter, of spatiotemporal variability. Our work lays a theoretical foundation for studying such networks and shows that they can exhibit rich dynamics, suggesting several directions for future study.

\section{ACKNOWLEDGMENTS}

This work was supported by NIH-1R01NS070865-01A1 and NSF-DMS-1313225. We thank Ashok Litwin-Kumar, Zachary Kilpatrick, Bard Ermentrout, and Jonathan Rubin for helpful discussions.

[1] F. Sagués, J. M. Sancho, and J. García-Ojalvo, Spatiotemporal Order Out of Noise, Rev. Mod. Phys. 79, 829 (2007).

[2] B. Lindner, J. Garcia-Ojalvo, A. Neiman, and L. Schimansky-Geier, Effects of Noise in Excitable Systems, Phys. Rep. 392, 321 (2004).

[3] R. B. Stein, E. R. Gossen, and K.E. Jones, Neuronal Variability: Noise or Part of the Signal?, Nat. Rev. Neurosci. 6, 389 (2005).

[4] G. Maimon and J. A. Assad, Beyond Poisson: Increased Spike-Time Regularity across Primate Parietal Cortex, Neuron 62, 426 (2009).

[5] M. M. Churchland, M. Byron, J.P. Cunningham, L.P. Sugrue, M. R. Cohen, G. S. Corrado, W. T. Newsome, A. M. Clark, P. Hosseini, B. B. Scott et al., Stimulus Onset Quenches Neural Variability: A Widespread Cortical Phenomenon, Nat. Neurosci. 13, 369 (2010).

[6] C. van Vreeswijk and H. Sompolinsky, Chaos in Neuronal Networks with Balanced Excitatory and Inhibitory Activity, Science 274, 1724 (1996).

[7] C. van Vreeswijk and H. Sompolinsky, Chaotic Balanced State in a Model of Cortical Circuits, Neural Comput. 10, 1321 (1998).

[8] N. Brunel, Dynamics of Sparsely Connected Networks of Excitatory and Inhibitory Spiking Neurons, J. Comput. Neurosci. 8, 183 (2000).

[9] A. Renart, N. Brunel, and X.-J. Wang, Computational Neuroscience: A Comprehensive Approach (CRC Press, Boca Raton, FL, 2004), pp. 431-490.

[10] S.E. Boustani and A. Destexhe, A Master Equation Formalism for Macroscopic Modeling of Asynchronous Irregular Activity States, Neural Comput. 21, 46 (2009).

[11] A. Renart, J. de La Rocha, P. Bartho, L. Hollender, N. Parga, A. Reyes, and K. Harris, The Asynchronous State in Cortical Circuits, Science 327, 587 (2010).

[12] K. Binder and A. P. Young, Spin Glasses: Experimental Facts, Theoretical Concepts, and Open Questions, Rev. Mod. Phys. 58, 801 (1986).

[13] Y. Shu, A. Hasenstaub, and D. A. McCormick, Turning On and Off Recurrent Balanced Cortical Activity, Nature (London) 423, 288 (2003).

[14] B. Haider, A. Duque, A. R. Hasenstaub, and D. A. McCormick, Neocortical Network Activity In Vivo Is Generated through a Dynamic Balance of Excitation and Inhibition, J. Neurosci. 26, 4535 (2006). 
[15] A. Ecker, P. Berens, G. Keliris, M. Bethge, N. Logothetis, and A. Tolias, Decorrelated Neuronal Firing in Cortical Microcircuits, Science 327, 584 (2010).

[16] M. London, A. Roth, L. Beeren, M. Häusser, and P. E. Latham, Sensitivity to Perturbations In Vivo Implies High Noise and Suggests Rate Coding in Cortex, Nature (London) 466, 123 (2010).

[17] S. Lim and M.S. Goldman, Balanced Cortical Microcircuitry for Maintaining Information in Working Memory, Nat. Neurosci. 16, 1306 (2013).

[18] M. Boerlin, C. K. Machens, and S. Denève, Predictive Coding of Dynamical Variables in Balanced Spiking Networks, PLoS Comput. Biol. 9, e1003258 (2013).

[19] D. Hansel and C. van Vreeswijk, The Mechanism of Orientation Selectivity in Primary Visual Cortex without a Functional Map, J. Neurosci. 32, 4049 (2012).

[20] A. Litwin-Kumar and B. Doiron, Slow Dynamics and High Variability in Balanced Cortical Networks with Clustered Connections, Nat. Neurosci. 15, 1498 (2012).

[21] C. Holmgren, T. Harkany, B. Svennenfors, and Y. Zilberter, Pyramidal Cell Communication within Local Networks in Layer 2/3 of Rat Neocortex, J. Physiol. 551, 139 (2003).

[22] A.-M. M. Oswald, B. Doiron, J. Rinzel, and A. D. Reyes, Spatial Profile and Differential Recruitment of GABAB Modulate Oscillatory Activity in Auditory Cortex, J. Neurosci. 29, 10321 (2009).

[23] H. Ko, S. B. Hofer, B. Pichler, K. A. Buchanan, P. J. Sjöström, and T. D. Mrsic-Flogel, Functional Specificity of Local Synaptic Connections in Neocortical Networks, Nature (London) 473, 87 (2011).

[24] R. B. Levy and A. D. Reyes, Spatial Profile of Excitatory and Inhibitory Synaptic Connectivity in Mouse Primary Auditory Cortex, J. Neurosci. 32, 5609 (2012).

[25] S. Coombes, Waves, Bumps, and Patterns in Neural Field Theories, Biol. Cybern. 93, 91 (2005).

[26] P. C. Bressloff and M. A. Webber, Front Propagation in Stochastic Neural Fields, SIAM J. Appl. Dyn. Syst. 11, 708 (2012).

[27] Z. P. Kilpatrick and B. Ermentrout, Wandering Bumps in Stochastic Neural Fields, SIAM J. Appl. Dyn. Syst. 12, 61 (2013).

[28] A. Hutt, A. Longtin, and L. Schimansky-Geier, Additive Noise-Induced Turing Transitions in Spatial Systems with Application to Neural Fields and the Swift-Hohenberg Equation, Physica (Amsterdam) 237D, 755 (2008).

[29] M. A. Buice, J. D. Cowan, and C. C. Chow, Systematic Fluctuation Expansion for Neural Network Activity Equations, Neural Comput. 22, 377 (2010).

[30] P. C. Bressloff, Stochastic Neural Field Theory and the System-Size Expansion, SIAM J. Appl. Math. 70, 1488 (2009).

[31] D. Somers, S. Nelson, and M. Sur, An Emergent Model of Orientation Selectivity in Cat Visual Cortical Simple Cells, J. Neurosci. 15, 5448 (1995).

[32] P. C. Bressloff, Spatiotemporal Dynamics of Continuum Neural Fields, J. Phys. A 45, 033001 (2012).

[33] M. N. Shadlen and W. T. Newsome, The Variable Discharge of Cortical Neurons: Implications for Connectivity, Computation, and Information Coding, J. Neurosci. 18, 3870 (1998).
[34] D. Amit and N. Brunel, Model of Global Spontaneous Activity and Local Structured Activity during Delay Periods in the Cerebral Cortex, Cereb. Cortex 7, 237 (1997).

[35] M. J. E. Richardson, Firing-Rate Response of Linear and Nonlinear Integrate-and-Fire Neurons to Modulated Current-Based and Conductance-Based Synaptic Drive, Phys. Rev. E 76, 021919 (2007).

[36] A. Politi, R. Livi, G.-L. Oppo, and R. Kapral, Unpredictable Behaviour in Stable Systems, Europhys. Lett. 22, 571 (1993).

[37] F. Ginelli, R. Livi, and A. Politi, Emergence of Chaotic Behaviour in Linearly Stable Systems, J. Phys. A 35, 499 (2002).

[38] T. P. Vogels and L. F. Abbott, Signal Propagation and Logic Gating in Networks of Integrate-and-Fire Neurons, J. Neurosci. 25, 10786 (2005).

[39] M. Monteforte and F. Wolf, Dynamic Flux Tubes form Reservoirs of Stability in Neuronal Circuits, Phys. Rev. X 2, 041007 (2012).

[40] E. Ledoux and N. Brunel, Dynamics of Networks of Excitatory and Inhibitory Neurons in Response to TimeDependent Inputs, Front. Comput. Neurosci. 5, 25 (2011).

[41] P. Landry and M. Deschênes, Intracortical Arborizations and Receptive Fields of Identified Ventrobasal Thalamocortical Afferents to the Primary Somatic Sensory Cortex in the Cat, J. Comp. Neurol. 199, 345 (1981).

[42] T. Freund, K. Martin, I. Soltesz, P. Somogyi, and D. Whitteridge, Arborisation Pattern and Postsynaptic Targets of Physiologically Identified Thalamocortical Afferents in Striate Cortex of the Macaque Monkey, J. Comp. Neurol. 289, 315 (1989).

[43] E. Rausell and E. Jones, Extent of Intracortical Arborization of Thalamocortical Axons as a Determinant of Representational Plasticity in Monkey Somatic Sensory Cortex, J. Neurosci. 15, 4270 (1995).

[44] R. Ben-Yishai, R. L. Bar-Or, and H. Sompolinsky, Theory of Orientation Tuning in Visual Cortex, Proc. Natl. Acad. Sci. U.S.A. 92, 3844 (1995).

[45] R. Shapley, M. Hawken, and D. L. Ringach, Dynamics of Orientation Selectivity in the Primary Visual Crtex and the Importance of Cortical Inhibition, Neuron 38, 689 (2003).

[46] F. G. Tricomi, Integral Equations (Interscience, New York, 1957).

[47] A. D. Polyanin and A. V. Manzhirov, Handbook of integral equations (CRC press, Boca Raton, FL, 2008).

[48] P. Hansen, Numerical Tools for Analysis and Solution of Fredholm Integral Equations of the First Kind, Inverse Probl. 8, 849 (1992).

[49] C. van Vreeswijk and H. Sompolinsky, in Irregular Activity in Large Networks of Neurons, Proceedings of the Les Houches Summer School, Course 9 C. Chow, B. Gutkin, D. Hansel, C. Meunier, and J. Dalibard (Elsevier, London, 2005), Vol. 80, pp. 341-406.

[50] Y. Roudi and P. E. Latham, A Balanced Memory Network, PLoS Comput. Biol. 3, e141 (2007).

[51] D. Hansel and G. Mato, Short-Term Plasticity Explains Irregular Persistent Activity in Working Memory Tasks, J. Neurosci. 33, 133 (2013). 